\title{
A new proof for the decidability of DOL ultimate periodicity
}

\author{
Vesa Halava $^{1}$, Tero Harju ${ }^{1}$ and Tomi Kärki ${ }^{1,2}$ \\ ${ }^{1}$ Department of Mathematics \\ University of Turku, 20014 Turku, Finland \\ ${ }^{2}$ Department of Teacher Education \\ University of Turku, PO Box 175, 26101 Rauma, Finland
}

\begin{abstract}
We give a new proof for the decidability of the DOL ultimate periodicity problem based on the decidability of $p$-periodicity of morphic words adapted to the approach of Harju and Linna.
\end{abstract}

\section{Introduction}

L systems were originally introduced by A. Lindenmayer to model the development of simple filamentous organisms [6, 7]. The challenging and fruitful study of these systems in the 70s and 80s created many new results and notions [9]. In this paper we consider the important problem of recognizing ultimately periodic DOL sequences.

Let $\mathscr{A}$ be a finite alphabet and denote the empty word by $\varepsilon$. A $D O L$ system is a pair $(h, u)$, where $h: \mathscr{A}^{*} \rightarrow \mathscr{A}^{*}$ is a morphism and $u$ is a finite word over $\mathscr{A}$. The language of the D0L system is $L(h, u)=$ $\left\{h^{i}(u) \mid i \geq 0\right\}$ and the limit set $\lim L(h, u)$ consists of all infinite words $w$ such that for all $n$ there is a prefix of $w$ longer than $n$ belonging to $L(h, u)$. Clearly, if the limit set is non-empty, then one can effectively find integers $p$ and $q$ such that $h^{p}(u)$ is a proper prefix of $h^{p+q}(u)$ and

$$
\lim L(h, u)=\bigcup_{i=0}^{q-1} \lim L\left(h^{q}, h^{p+i}(u)\right),
$$

where $\left|\lim L\left(h^{q}, h^{p+i}(u)\right)\right|=1$. Hence, we may restrict to D0L systems $(h, u)$ where $h$ is prolongable on $u$, i.e., $h(u)=u y$ and $h^{n}(y) \neq \varepsilon$ for all integers $n \geq 0$. In this case, $h^{n}(u)$ is a prefix of $h^{n+1}(u)$ and the limit is the following fixed point of $h$ :

$$
h^{\omega}(u)=\lim _{n \rightarrow \infty} h^{n}(u)=u y h(y) h^{2}(y) \cdots
$$

An infinite word $x$ is ultimately periodic if it is of the form $x=u v^{\omega}=u v v v \cdots$, where $u$ and $v$ are finite words. The length $|u|$ is a preperiod and the length $|v|$ is a period of $x$. An infinite word $x$ is ultimately p-periodic if $|v|=p$. The smallest period of $x$ is called the period of $x$.

Now we are ready to formulate the DOL ultimate periodicity problem: Given a morphism $h$ prolongable on $u$, decide whether $h^{\omega}(u)$ is ultimately periodic. Note that in this problem we may assume that $u$ is a letter. Indeed, if $h(u)=u y$, then instead of $(h, u)$ we may consider $\left(h^{\prime}, a\right)$ where $a \notin \mathscr{A}$ and $h^{\prime}:(\mathscr{A} \cup\{a\})^{*} \rightarrow(\mathscr{A} \cup\{a\})^{*}$ where $h^{\prime}(a)=a y$ and $h^{\prime}(b)=h(b)$ for every $b \in \mathscr{A}$. The limit $h^{\omega}(u)$ is ultimately periodic if and only if $h^{\prime \omega}(a)$ is.

The decidability of the ultimate periodicity question for DOL sequences was proven by T. Harju and M. Linna [4] and, independently, by J.-J. Pansiot [8]; see also a more recent proof of J. Honkala [5]. In

P. Ambrož, Š. Holub and Z. Masáková (Eds.):

8th International Conference WORDS 2011

EPTCS 63, 2011, pp. 147-151 doi 10.4204/EPTCS.63.20 
the binary case the problem was effectively solved by Séébold [10]. Here we show how the proof of [4] can be simplified using a recent result concerning the decidability of the $p$-periodicity problem.

Before giving the proof, we introduce the following notation. Given a morphism $h: \mathscr{A}^{*} \rightarrow \mathscr{A}^{*}$, we call a letter $b \in \mathscr{A}$ finite if $\left\{h^{n}(b) \mid n \geq 0\right\}$ is a finite set. Otherwise, $b$ is an infinite letter. Moreover, we say that a letter $b$ is recurrent in $h^{\omega}(a)$ if it occurs infinitely often in $h^{\omega}(a)$. For a given morphism $h$ prolongable on $a$ and for an infinite word $h^{\omega}(a)$, denote the set of finite letters by $\mathscr{A}_{F}$, the set on infinite letters by $\mathscr{A}_{I}$ and the set of recurrent letters by $\mathscr{A}_{R}$. Also, denote by $\mathscr{A}_{1}$ the subset of $\mathscr{A}$ which consists of the infinite letters occurring infinitely many times in $h^{\omega}(a)$, i.e., $\mathscr{A}_{1}=\mathscr{A}_{I} \cap \mathscr{A}_{R}$.

Let us shortly describe how the sets $\mathscr{A}_{F}, \mathscr{A}_{I}$ and $\mathscr{A}_{R}$ can be constructed. Note that if $b$ is a mortal letter, i.e., $h^{n}(b)=\varepsilon$ for some $n \geq 1$, then $h^{|\mathscr{A}|}(b)=\varepsilon$. Denote $\hat{h}=h^{|\mathscr{A}|}$ and denote the set of the mortal letters by $\mathscr{M}$. Note also that $b$ is a finite letter if and only if there exists a word $u \in\left\{h^{n}(b) \mid n \geq 0\right\}$ such that $u=h^{p}(u)$ for some $p \geq 1$. Clearly, $\left\{\hat{h}^{n}(b) \mid n \geq 0\right\}$ is finite if and only if $\left\{h^{n}(b) \mid n \geq 0\right\}$ is finite. Hence, by replacing $h$ with $\hat{h}$ we may assume that $h(b)=\varepsilon$ if $b \in \mathscr{M}$. Moreover, let $\mathscr{B}=\mathscr{A} \backslash \mathscr{M}$ and let $g: \mathscr{B}^{*} \rightarrow \mathscr{B}^{*}$ be a morphism defined by $g(b)=\mu h(b)$, where

$$
\mu(b)= \begin{cases}\varepsilon, & \text { if } b \in \mathscr{M}, \\ b, & \text { otherwise. }\end{cases}
$$

Now $g$ is non-erasing, and $b \in \mathscr{A}_{F}$ if and only if $\left\{g^{n}(b) \mid n \geq 0\right\}$ is finite. Namely, for any $n \geq 0$, we know by the definition of $g$ that the word $h^{n}(b)$ can be obtained by inserting a finite number of mortal letters to $g^{n}(b)$. The set $\left\{g^{n}(b) \mid n \geq 0\right\}$ is finite if and only if for some $n$ all letters in $g^{n}(b)$ belong to $U_{1}=\left\{b \in \mathscr{B} \mid g^{i}(b) \in \mathscr{B}\right.$ for every $\left.i \geq 0\right\}$. If $U_{i}=\left\{b \in \mathscr{B} \mid g(b) \in U_{i-1}^{*}\right\}$, then $U_{i-1} \subseteq U_{i}$ and

$$
\mathscr{A}_{F} \backslash \mathscr{M}=\bigcup_{i=1}^{\infty} U_{i}=U_{|\mathscr{A}|} .
$$

Hence, we can effectively calculate $\mathscr{A}_{F}$ and $\mathscr{A}_{I}=\mathscr{A} \backslash \mathscr{A}_{F}$. In order to find the recursive letters, we construct a graph $G$ where the set of vertices is $\mathscr{A}$ and there is an edge from $b$ to $c$ if $c$ occurs in the image $h(b)$. Let $h(a)=a x$. If there are infinitely many paths from a letter in $x$ to the letter $b$, then $b$ occurs infinitely many times in $h^{\omega}(a)$.

\section{Decidability of the $p$-periodicity problem}

Let $p \geq 1$, and let $x=\left(x_{n}\right)_{n \geq 0}$ be an infinite word over $\mathscr{A}=\left\{a_{1}, \ldots, a_{d}\right\}$. For $0 \leq k \leq p-1$, we say that the letters occurring infinitely many times in positions $x_{n}$, where $n \equiv k(\bmod p)$, form the $k$-set of $x$ modulo $p$. It was shown in [3] that these $k$-sets can be effectively constructed for $x=h^{\omega}(u)$, where $h$ is prolongable on the word $u$. This is based on the fact that there exist integers $r$ and $q$ such that

$$
\left|h^{r}(b)\right| \equiv\left|h^{r+q}(b)\right| \quad(\bmod p)
$$

for every letter $b \in \mathscr{A}$. The incidence matrix of $h$ is the matrix $M=\left(m_{i, j}\right)_{1 \leq i, j \leq d}$ where $m_{i, j}$ denotes the number of occurrences of $a_{i}$ in $h\left(a_{j}\right)$. The sequence of matrices $M^{n} \bmod p$, where the entries are the residues modulo $p$, must be ultimately periodic. Since $\left|h^{n}\left(a_{j}\right)\right|(\bmod p)$ is the sum of the elements in the $j$ th column of $M^{n}$, we conclude that the sequence $\left(\left|h^{n}\left(a_{j}\right)\right|\right)_{n \geq 0}(\bmod p)$ is ultimately periodic for every $a_{j} \in \mathscr{A}$ and (1) follows.

In order to find the $k$-sets of $x$ modulo $p$ we construct a directed graph $G_{h}=(V, E)$ where the set of vertices $V$ is $\{(a, i) \mid a \in \mathscr{A}, 0 \leq i<p\}$ and there is an edge from $(c, i)$ to $(d, j)$ if, for some $b$ in $x$, the 


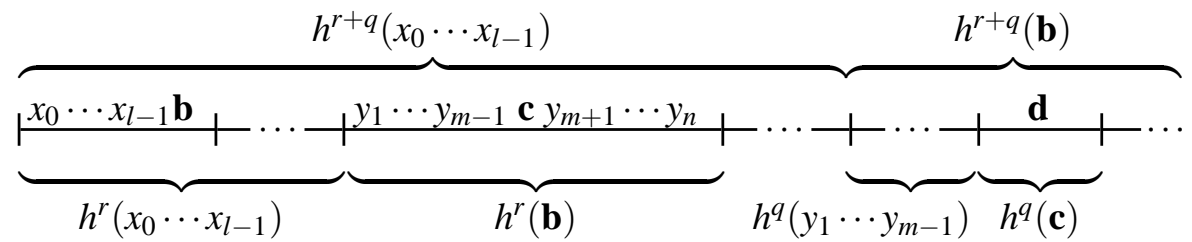

Figure 1: Images $h^{r}(b)$ and $h^{r+q}(b)$.

letter $c$ occurs in the image $h^{r}(b)$ at position congruent to $i(\bmod p)$ in $x$, and the letter $d$ occurs in the image $h^{q}(c)$ at position congruent to $j(\bmod p)$ in $x$; see Figure 1

It is possible to construct such a graph by calculating the images $h^{r}(b)$ and $h^{r+q}(b)$ for every $b \in \mathscr{A}$. Namely, if $b=x_{l}$ and $c$ is the $m$ th letter of $h^{r}(b)=y_{1} \cdots y_{n}$ and $d$ is the $m^{\prime}$ th letter of $h^{q}(c)$, then we have

$$
\begin{aligned}
i & \equiv\left|h^{r}\left(x_{0} \cdots x_{l-1}\right)\right|+m-1 \quad(\bmod p), \\
j & \equiv\left|h^{r+q}\left(x_{0} \cdots x_{l-1}\right)\right|+\left|h^{q}\left(y_{1} \cdots y_{m-1}\right)\right|+m^{\prime}-1 \quad(\bmod p) .
\end{aligned}
$$

By (1), we have $\left|h^{r+q}\left(x_{0} \cdots x_{l-1}\right)\right| \equiv\left|h^{r}\left(x_{0} \cdots x_{l-1}\right)\right|(\bmod p)$, which together with (2) and (3) implies

$$
j \equiv\left|h^{q}\left(y_{1} \cdots y_{m-1}\right)\right|+i+m^{\prime}-m \quad(\bmod p) .
$$

We say that a vertex $(c, i) \in V$ is an initial vertex if there exists a letter $b=x_{l}$ such that $0 \leq l<\left|h^{r}(a)\right|, c$ is the $m$ th letter of $h^{r}(b)$ and $i$ satisfies (2). A vertex $(c, k)$ is called recurrent if there exist infinitely many paths starting from some initial vertex and ending in $(c, k)$. By construction, this means that $c$ belongs to the $k$ set of $x$ modulo $p$.

Given a coding $g$ and a morphism $h: \mathscr{A}^{*} \rightarrow \mathscr{A}^{*}$ prolongable on $a$, it is easy to see that the morphic word $g\left(h^{\omega}(a)\right)$ is ultimately $p$-periodic if and only if $g(b)=g(c)$ for all pairs of letters $(b, c)$ such that $b$ and $c$ belong to the same $k$-set of $h^{\omega}(a)$ modulo $p$. Since the $k$-sets of $h^{\omega}(a)$ can be effectively constructed, we have the following result proved in [3].

Theorem 1. Given a positive integer $p$, it is decidable whether a morphic word $g\left(h^{\omega}(a)\right)$ is ultimately p-periodic.

\section{Decidability of the DOL ultimate periodicity problem}

Before the decidability proof, we give the following result proved in [1, 2]; see also [5].

Theorem 2. Let $h: \mathscr{A}^{*} \rightarrow \mathscr{A}^{*}$ be a morphism and $u, v \in \mathscr{A}^{*}$. If there is a positive integer $n$ such that $h^{n}(u)=h^{n}(v)$, then $h^{|\mathscr{A}|}(u)=h^{|\mathscr{A}|}(v)$.

This theorem can be proved by induction on the size of the alphabet and the induction step is based on elementary morphisms. A morphism $h: \mathscr{A}^{*} \rightarrow \mathscr{A}^{*}$ is called elementary if there do not exist an alphabet $\mathscr{B}$ smaller than $\mathscr{A}$ and two morphisms $f: \mathscr{A}^{*} \rightarrow \mathscr{B}^{*}$ and $g: \mathscr{B}^{*} \rightarrow \mathscr{A}^{*}$ such that $h=g f$. 
Since elementary morphisms are injective, the claim is clear if $h$ is elementary. Now assume that $h=g f$ as above. Then $h^{n}(u)=h^{n}(v)$ implies that $(f g)^{n} f(u)=(f g)^{n} f(v)$ and, by induction, $(f g)^{|\mathscr{B}|} f(u)=$ $(f g)^{|\mathscr{B}|} f(v)$. This proves the claim, since $(g f)^{|\mathscr{B}|+1}(u)=(g f)^{|\mathscr{B}|+1}(v)$ and $|\mathscr{A}| \geq|\mathscr{B}|+1$.

Using Theorem 1 and Theorem 2 and following the guidelines in [4] we give a new proof for the decidability of the DOL ultimate periodicity problem. The difference between the original proof of Harju and Linna and this proof is that we employ a new method obtained from $p$-periodicity as stated in Theorem 1

Theorem 3. The ultimate periodicity problem is decidable for DOL sequences.

Proof. As explained above, it suffices to show that we can decide whether $h^{\omega}(a)$ is ultimately periodic for a given morphism $h: \mathscr{A}^{*} \rightarrow \mathscr{A}^{*}$ prolongable on $a$. Without loss of generality, we assume that every letter of $\mathscr{A}$ really occurs in $h^{\omega}(a)$. Otherwise, we could consider a restriction of $h$. Recall also that $\mathscr{A}_{1}$ is the subset of $\mathscr{A}$ which consists of the infinite letters occurring infinitely many times in $h^{\omega}(a)$.

If $\mathscr{A}_{1}=\emptyset$, then the sequence is ultimately periodic. Namely, if $h(a)=a y$ and $y$ contains infinite letters, then every image $h^{n}(y)$ contains infinite letters and there must be at least one infinite letter occurring infinitely many times in $h^{\omega}(a)=a y h(y) h^{2}(y) \cdots$, which means that $\mathscr{A}_{1} \neq \emptyset$. Therefore, there is only one infinite letter and it is the letter $a$ occurring once in the beginning of the word. Hence, $h(a)=a y$ where $y$ consists of finite letters. Then there must be integers $n$ and $p$ such that $h^{n+p}(y)=h^{n}(y)$. Thus $\left|h^{n}(y) h^{n+1}(y) \cdots h^{n+p-1}(y)\right|$ is a period of $h^{\omega}(a)$.

Assume now that $b \in \mathscr{A}_{1}$. We may write

$$
h^{\omega}(a)=u_{0} b u_{1} b u_{2} \cdots,
$$

where $u_{i} \in(\mathscr{A} \backslash\{b\})^{*}$. If the set $U=\left\{u_{i} \mid i \geq 0\right\}$ is infinite then $h^{\omega}(a)$ cannot be ultimately periodic. Note that if there exists a $c \in \mathscr{A}_{I}$ such that the letter $b$ does not occur in any $h^{i}(c)$, then $U$ is infinite. This property is clearly decidable since if a letter occurs in $h^{i}(c)$ for some $i$, then it occurs in the image for $i \leq|\mathscr{A}|$. Hence, we may assume that for each infinite letter $c$ the letter $b$ occurs in $h^{i}(c)$ for some $i \leq|\mathscr{A}|$.

Next we show that we may decide if $U$ is infinite or not. First assume that $U$ is infinite. Then there are arbitrarily long words in $U$. Since each infinite letter from $h^{\omega}(a)$ produces an occurrence of $b$ in at most $|\mathscr{A}|$ steps, there must be arbitrarily long words from $\mathscr{A}_{F}$ in $U$. This is possible only if for some $c \in \mathscr{A}_{I}$ and integer $s \leq|\mathscr{A}|$ we have $h^{s}(c)=v_{1} c v_{2}$, where for $i=1$ or $i=2$ we have $v_{i} \in \mathscr{A}_{F}^{+}$and $h^{n}\left(v_{i}\right) \neq \varepsilon$ for every $n \geq 0$. This is a property that we can effectively check. Note that if $h^{n}\left(v_{i}\right)=\varepsilon$ for some $n \geq 0$, then $h^{|\mathscr{A}|}\left(v_{i}\right)=\varepsilon$. On the other hand, if there exists $c \in \mathscr{A}_{I}$ satisfying the above conditions, the set $U$ is clearly infinite. Hence, the finiteness of $U$ can be verified and the finite set $U$ can be effectively constructed.

Now assume that $h^{\omega}(a)$ is ultimately periodic, i.e., $h^{\omega}(a)=u v^{\omega}$, where $v$ is primitive. Consider a subset $U^{\prime}$ of $U$ containing the elements $u_{i}$ occurring infinitely many times in $h^{\omega}(a)$. Since $b$ is in $\mathscr{A}_{I}$, there exists an integer $N$ such that $\left|h^{n}(b)\right| \geq|v|$ for every $n \geq N$. Hence, let $n \geq N$. Since $b u_{i}$ with $u_{i} \in U^{\prime}$ occurs in the periodic part of the sequence, we conclude that $h^{n}\left(b u_{i}\right) \in w_{n} \mathscr{A}^{*}$, where $w_{n}$ is a conjugate of $v$. Moreover, by the primitivity of $v$ and $w_{n}$, we have

$$
h^{n}\left(b u_{i}\right) \in w_{n}^{*} \quad \text { for all } u_{i} \in U^{\prime} .
$$

Namely, assume that $h^{n}\left(b u_{i}\right)=w_{n}^{t} w^{\prime}$, where $t$ is some positive integer and $w^{\prime}$ is a proper prefix of $w_{n}$, i.e., $w^{\prime}$ is non-empty and $w^{\prime} \neq w_{n}$. Then $h^{n}\left(b u_{i} b\right) \in w_{n}^{t} w^{\prime} w_{n} \mathscr{A}^{*}$ is a prefix of $w_{n}^{\omega}$, which implies that the word $w_{n}$ occurring after $w^{\prime}$ occurs inside $w_{n}^{2}$. Since $w_{n}$ is primitive, this is impossible. 
Take now any two words $u_{i}$ and $u_{j} \in U^{\prime}$. By (4), we conclude that there exists $m$ such that $h^{\ell}\left(b u_{i} b u_{j}\right)=$ $h^{\ell}\left(b u_{j} b u_{i}\right)$ for all $\ell \geq m$. Moreover, by Theorem 2 , we know that we may choose $m=|\mathscr{A}|$. Note that if the above does not hold for some $u_{i}$ and $u_{j}$ in $U^{\prime}$, then $h^{\omega}(a)$ cannot be ultimately periodic. Hence, let $m=|\mathscr{A}|$ and

$$
h^{m}\left(b u_{i} b u_{j}\right)=h^{m}\left(b u_{j} b u_{i}\right),
$$

for every $u_{i}, u_{j} \in U^{\prime}$. Then the words $h^{m}\left(b u_{i}\right)$ and $h^{m}\left(b u_{j}\right)$ commute and by transitivity we can find a primitive word $z$ such that

$$
h^{\ell}\left(b u_{i}\right) \in z^{*} \text { for all } u_{i} \in U^{\prime}, \ell \geq m \text {. }
$$

This implies that $h^{\omega}(a)$ is ultimately $|z|$-periodic. Since we can test the ultimate $|z|$-periodicity of $h^{\omega}(a)$ by Theorem 11, the ultimate periodicity problem of $h^{\omega}(a)$ is decidable.

\section{References}

[1] K. Culik, II (1978): The decidability of v-local catenativity and of other properties of D0L systems. Information Processing Lett. 7(1), pp. 33-35, doi:10.1016/0020-0190 (78)90035-2.

[2] A. Ehrenfeucht \& G. Rozenberg (1978): Simplifications of homomorphisms. Inform. and Control 38(3), pp. 298-309, doi:10.1016/S0019-9958(78) 90095-5.

[3] V. Halava, T. Harju, T. Kärki \& M. Rigo (2010): On the periodicity of morphic words. In Y. Gao, H. Lu, S. Seki \& S. Yu, editors: Developments in Language Theory, 14th International Conference, DLT 2010, London, ON, Canada, August 17-20, 2010. Proceedings, Lecture Notes in Comput. Sci. 6224, Springer, Berlin, pp. 209-217, doi:10.1007/978-3-642-14455-4_20.

[4] T. Harju \& M. Linna (1986): On the periodicity of morphisms on free monoids. RAIRO Inform. Théor. Appl. 20(1), pp. 47-54.

[5] J. Honkala (2008): Cancellation and periodicity properties of iterated morphisms. Theoret. Comput. Sci. 391(1-2), pp. 61-64, doi:10.1016/j.tcs.2007.10.030.

[6] A. Lindenmayer (1968): Mathematical models for cellular interactions in development I. Filaments with one-sided inputs. J. Theoret. Biol. 18, pp. 280-299, doi:10.1016/0022-5193(68)90079-9.

[7] A. Lindenmayer (1968): Mathematical models for cellular interactions in development II. Simple and branching filaments with two-sided inputs. J. Theoret. Biol. 18, pp. 300-315, doi:10.1016/ 0022-5193(68) 90080-5.

[8] J.-J. Pansiot (1986): Decidability of periodicity for infinite words. RAIRO Inform. Théor. Appl. 20(1), pp. 43-46.

[9] G. Rozenberg \& A. Salomaa (1986): The Book of L. Springer, Berlin.

[10] P. Séébold (1988): An effective solution to the D0L-periodicity problem in the binary case. EATCS Bull. 36, pp. 137-151. 